\title{
DIAGNÓSTICOS DE ENFERMAGEM EM CLIENTES COM ALTERAÇÕES HEMATOLÓGICAS: USO DA TAXONOMIA I DA NANDA
}

Cristine Alves Costa de Jesus*

Emília Campos de Carvalho **

JESUS, C.A.C. de; CARVALHO, E.C. de. Diagnósticos de enfermagem em clientes com alterações hematológicas: uso da Taxonomia I da NANDA. Rev.latino-am.enfermagem, Ribeirão Preto, v. 5, n. 4, p. 91-99, outubro 1997.

O presente estudo tem por objetivo descrever os diagnósticos de enfermagem mais freqüentes encontrados em um grupo de clientes com alterações hematológicas. Utilizou-se a Taxonomia I, proposta pela North American Nursing Diagnosis (NANDA), para o direcionamento da pesquisa. A amostra compreendeu 14 clientes, sendo identificados 16 diagnósticos de enfermagem com freqüência igual ou superior a 50,0\%. Recomenda-se a realização de outros estudos para melhor definição do perfil diagnóstico dessa clientela.

UNITERMOS: diagnóstico de enfermagem, processo de enfermagem, cliente com alterações hematológicas

\section{INTRODUÇÃO}

A Enfermagem vem, há décadas, em busca da organização do conhecimento científico específico, expresso inicialmente na forma do plano de cuidados. Inúmeras discussões quanto a necessidade de operacionalização dos referenciais teóricos de enfermagem, bem como os avanços em pesquisas fizeram surgir, na década de setenta, o processo de enfermagem.

As primeiras definições de processo de enfermagem tiveram como cerne o inter-relacionamento enfermeiro/cliente. Atualmente é concebido como um método sistemático de conduzir a prática de enfermagem, a partir da aplicação do método científico - avaliação inicial do estado de saúde do cliente, identificação de seus problemas atuais ou potenciais sob a competência do enfermeiro, por intermédio do julgamento clínico, formulação de critérios de resultado e estratégias, planejamento, implementação e avaliação dos resultados estabelecidos a nível individual ${ }^{9}$.

São descritas cinco etapas do processo de enfermagem: a coleta de dados, o diagnóstico, o planejamento, a implementação e a avaliação ${ }^{17}$.

Dentre elas, a que tem merecido maior destaque é a análise e interpretação dos dados coletados, chamada de diagnóstico de enfermagem. Tem sido considerada não apenas uma etapa isolada de descrição de problemas, mas que promove a integração da coleta de dados ao planejamento das ações, envolvendo julgamento, avaliação crítica e tomada de decisão ${ }^{1,16}$.

Desde 1973, quando Kristine Gebbie e Mary Ann Lavin organizaram a primeira Conferência de Classificação dos Diagnósticos de Enfermagem na Universidade de St. Louis, o diagnóstico de enfermagem passou a ser visto como uma etapa de destaque, dinâmica, sistemática, organizada e complexa do processo de enfermagem ${ }^{8}$.

A necessidade de se identificar, organizar e classificar os diagnósticos de enfermagem, com base em regras consistentes, e ainda, de criar uma Taxonomia dos diagnósticos de enfermagem, fez surgir, nos Estados Unidos, a Associação Norte Americana dos Diagnósticos de Enfermagem (NANDA), que, segundo THOMAS \& NEWSOME $^{19}$, tem desenvolvido um dos sistemas de classificação dos diagnósticos de enfermagem mais aceitos e conhecidos atualmente.

Diagnóstico de enfermagem, segundo NANDA, é “(...) um julgamento clínico das respostas do indivíduo, da família ou da comunidade aos processos vitais ou aos problemas de saúde atuais ou potenciais. Os diagnósticos de enfermagem fornecem a base para a seleção das intervenções de enfermagem, para atingir resultados, pelos quais o enfermeiro é responsável"2.

Os diagnósticos de enfermagem propostos pela NANDA têm, em última análise, a finalidade de fornecer embasamento científico à prática do cuidar; prática essa

\footnotetext{
* Professora Assistente do Departamento de Enfermagem da Universidade de Brasília. Pós-graduanda da Área de Enfermagem Fundamental - Nível Doutorado - Escola de Enfermagem de Ribeirão Preto - USP

** Professora Titular da Escola de Enfermagem de Ribeirão Preto da Universidade de São Paulo
} 
que tradicionalmente tem significado "discriminar situações que necessitem de intervenções de enfermagem",

Tem se considerado fundamental o reconhecimento e análise do diagnóstico, para a elaboração orientada do plano terapêutico e a instituição favorável das intervenções de enfermagem ${ }^{5,9,18}$.

O estudo de um grupo de clientes com características em comum (como a dos portadores de alterações hematológicas) proporcionará a identificação dos diagnósticos de enfermagem, suas causas e expressões. Além disso, irá favorecer a identificação de estratégias para elevar a qualidade da assistência e contribuir na formação de recursos humanos. "Diagnósticos de enfermagem comuns a um grupo de indivíduos delineiam o perfil deste grupo, permitindo um direcionamento global das intervenções de enfermagem" .

No que se refere ao portador de doença hematológica, é fundamental que sejam considerados os vários aspectos envolvidos, a exemplo: alterações físicas, psicológicas, emocionais e comportamentais.

A doença hematológica é na maioria dos casos crônica e provoca incapacidades variadas caracterizadas pelo declínio lento e progressivo da fisiologia normal ${ }^{3}$.

O portador de alterações hematológicas é um "ser total" que convive com o impacto das perdas tanto de natureza biológica, quanto psicológica e sociocultural. Podem advir conflitos emocionais significativos ligados a ameaças de alterações na imagem corporal e na autoimagem, perda de identidade, sentimentos de angústia, medo, depressão ou raiva, mudanças de papéis, isolamento social, dependência física e psicológica, dentre outros, que geram desequilíbrios e dificuldades de adaptações.

Além dos distúrbios emocionais os clientes hematológicos podem apresentar sinais e sintomas variados, decorrentes de alterações em diversos sistemas orgânicos. As modificações na crase sangüínea, presentes nos portadores de doença hematológica, geram distúrbios na oxigenação, coagulação e sistema de defesa do organismo, provocando muitas vezes fraqueza, debilidade física, emagrecimento, febre, lesões ulcerativas de pele e mucosas, parestesias, dores, sangramentos e infecções ${ }^{9}$.

Considerando que a sistematização da assistência de enfermagem conduz a ações fundamentadas e individualizadas, propõe-se, neste estudo, a identificação dos diagnósticos de enfermagem mais freqüentes dirigidos a um grupo de clientes com alterações hematológicas, conforme a classificação da Associação Norte Americana dos Diagnósticos de Enfermagem (North American Nursing Diagnosis Association NANDA) ${ }^{15}$.

\section{METODOLOGIA}

$\mathrm{O}$ estudo foi realizado com clientes internados no Setor de Hematologia de um hospital escola de um município do Estado de São Paulo, durante o período de setembro a novembro de 1991, e os dados aqui apresentados são oriundos de uma pesquisa mais abrangente, JESUS $^{9}$, que teve por finalidade analisar a aplicação do processo de enfermagem, baseado no referencial teórico de Imogene King.

Fizeram parte da amostra 14 clientes internados no referido período, que foram selecionados a partir dos seguintes critérios: internação motivada por alterações hematológicas, idade mínima de 14 anos, capacidade de interação preservada e anuência, por escrito, em participar do estudo.

Obedeceu-se a sequiência das etapas do processo de enfermagem, que inclui a coleta de dados, a formulação do diagnóstico, o planejamento, a implementação e a avaliação.

A coleta de dados foi realizada por uma das autoras, após esclarecimentos ao cliente quanto à finalidade da pesquisa e obtenção de sua anuência. Foram coletados dados a partir da entrevista, exame físico e observação, utilizando-se para o registro um instrumento, fundamentado no referencial teórico de Imogene King, previamente validado em estudo piloto.

A análise dos dados, identificação de problemas e a formulação dos diagnósticos de enfermagem foram realizados pela mesma autora que procedeu a coleta dos dados, e em um encontro seguinte com o cliente, estes eram discutidos a fim de se confirmar os problemas percebidos por ambos.

Foram estabelecidos os diagnósticos de enfermagem obedecendo-se a Taxonomia I da NANDA ${ }^{15}$. As demais etapas do processo (planejamento, implementação e avaliação) não serão consideradas no âmbito deste estudo.

Além disso, foram identificadas as variáveis sexo e idade com o propósito de caracterizar a amostra estudada.

\section{RESULTADOS E DISCUSSÃO}

Os resultados estão apresentados de acordo com o objetivo proposto. Inicialmente estão apontados os dados de caracterização da população do estudo e a seguir os diagnósticos de enfermagem.

Conforme pode ser visto na Tabela 1 , dos 14 clientes estudados, oito $(57,17 \%)$ pertenciam ao sexo masculino e seis $(42,88 \%)$ ao feminino. A idade variou entre 14 a 84 anos. 
Tabela 1 - Distribuição dos clientes estudados segundo faixa etária e sexo

\begin{tabular}{|c|c|c|c|c|c|c|}
\hline \multirow[t]{2}{*}{ Sexo } & \multicolumn{2}{|c|}{ Masculino } & \multicolumn{2}{|c|}{ Feminino } & \multicolumn{2}{|c|}{ Total } \\
\hline & $\mathbf{n}^{0}$ & $\%$ & $\mathbf{n}^{0}$ & $\%$ & $\mathbf{n}^{0}$ & $\%$ \\
\hline 1417 & - & - & 02 & 14,30 & 02 & 14,30 \\
\hline $18-59$ & 06 & 42,87 & 03 & 21,44 & 09 & 64,30 \\
\hline $60-84$ & 02 & 14,30 & 01 & 7,14 & 03 & 21,44 \\
\hline TOTAL & 08 & 57,17 & 06 & 42,88 & 14 & 100,0 \\
\hline
\end{tabular}

Os dados revelam que, quanto à variável sexo, houve uma distribuição ligeiramente maior para o sexo masculino $(57,17 \%)$ comparado ao sexo feminino $(42,88 \%)$, na população estudada. Quanto à variável idade, conforme se observa na Tabela 1, houve uma distribuição predominante para a faixa etária classificada de 18 a 59 anos. Estes dados relativos às variáveis sexo e idade, vão ao encontro dos dados de literatura quanto à distribuição epidemiológica das doenças hematológicas.

Embora não tenha sido interesse do estudo a identificação dos diagnósticos médicos, cabe esclarecer que fizeram parte da amostra clientes com Linfomas, Leucemias, Anemias e Mieloma, isoladamente. Todas essas alterações hematológicas têm distribuições variadas, acometendo ambos os sexos e ampla faixa etária.

Cabe ressaltar que a grande maioria da amostra, 09 clientes $(64,30 \%)$, foi constituída por adultos, indivíduos entre as faixas etárias apontadas dos 18 aos 59 anos, cuja fase de vida é identificada como a de maior produção, isto pode influenciar a forma do cliente reagir à doença e à hospitalização ${ }^{11}$.

Muitas vezes conflitos decorrentes da mudança no estilo de vida, crise situacional, ansiedade, revolta e medo, poderão estar presentes, caracterizando a importância da avaliação dos aspectos ligados ao processo de crescimento e desenvolvimento humanos.

No que se refere aos diagnósticos de enfermagem, a freqüência média foi de 16,9 diagnósticos para cada cliente.

À época, dos 99 diagnósticos apresentados na Taxonomia I da NANDA ${ }^{15}$, foram detectados 50 diagnósticos na amostra estudada, conforme mostra a Tabela 2. Para fins deste estudo serão discutidos apenas os diagnósticos que obtiveram frequiências iguais ou superiores à $50 \%$.
Tabela 2 - Distribuição dos diagnósticos de enfermagem na amostra, segundo sua frequiência

\begin{tabular}{|c|c|c|}
\hline \multirow[t]{2}{*}{ Diag nósticos de Enfermagem } & \multicolumn{2}{|c|}{ Frequência } \\
\hline & & $\%$ \\
\hline 01. Proteção alterada & 14 & 100,0 \\
\hline 02. Déficit de conhecimento & 14 & 100,0 \\
\hline 03. Alto risco para infecção & 12 & 85,7 \\
\hline 04. Integridade da pele prejudicada & 10 & 71,4 \\
\hline 05. Senso-percepção alterada & 10 & 71,4 \\
\hline 06. Distúrbio no padrão do sono & 10 & 71,4 \\
\hline 07. Dor & 10 & 71,4 \\
\hline 08. Alto risco para ferimentos & 09 & 64,3 \\
\hline 09. Excesso do volume de líquidos & 09 & 64,3 \\
\hline $\begin{array}{l}\text { 10. Estratégias ineficazes de resolução } \\
\text { individual }\end{array}$ & 08 & 57,1 \\
\hline $\begin{array}{l}\text { 11. Nutrição alterada: ingestão menor que as } \\
\text { necessidades comporais }\end{array}$ & 08 & 57,1 \\
\hline 12. Mucosa oral alterada & 07 & 50,0 \\
\hline 13. Ansiedade & 07 & 50,0 \\
\hline 14. Medo & 07 & 50,0 \\
\hline 15. Intolerância à atividade & 07 & 50,0 \\
\hline 16. Déficit & 07 & 50,0 \\
\hline $\begin{array}{l}\text { 17. Alto risco para prejuizo na integridade da } \\
\text { pele }\end{array}$ & 06 & 42,6 \\
\hline 18. Mobilidade fisica prejudicada & 06 & 42,6 \\
\hline 19. Fadiga & 06 & 42,6 \\
\hline 20. Distúrbio da imagem corporal & 05 & 35,7 \\
\hline 21. Déficit no auto-cuidado: higiene corporal & 05 & 35,7 \\
\hline $\begin{array}{l}\text { 22. Alto risco para alteração da temperatura } \\
\text { corporal }\end{array}$ & 04 & 28,6 \\
\hline 23. Hipertermia & 04 & 28,6 \\
\hline 24. Integridade tissular prejudicada & 04 & 28,6 \\
\hline 25. Negação & 04 & 28,6 \\
\hline 26. Eliminação urinária alterada & 04 & 28,6 \\
\hline $\begin{array}{l}\text { 27. Déficit no auto-cuidado: vestir-se e/ou } \\
\text { arrumar-se }\end{array}$ & 04 & 28,6 \\
\hline 28. Alto risco para trauma & 03 & 21,4 \\
\hline para sindrome do desuso & 03 & 21,4 \\
\hline 30. Manutenção da saúde alterada & 03 & 21,4 \\
\hline 31. Desempenho de papel alterado & 03 & 21,4 \\
\hline 32. Disfunção sexual & 03 & 21,4 \\
\hline 33. Eliminação traqueobrônquica ineficaz & 03 & 21,4 \\
\hline 34. Deglutição prejudicada & 03 & 21,4 \\
\hline 35. Desesperança & 02 & 14,3 \\
\hline 36. Imp otência & 02 & 14,3 \\
\hline 37. Disfunção na reação de pes & 02 & 14,3 \\
\hline $\begin{array}{l}\text { 38. Estratégias ineficazes de resolução familiar } \\
\text { comprometedoras }\end{array}$ & 02 & 14,3 \\
\hline 39. Constipação & 02 & 14,3 \\
\hline 40. Constipação colônica & 02 & 14,3 \\
\hline 41. Padrão respiratório ineficaz & 02 & 14,3 \\
\hline 42. Adaptação prejudicada & 01 & 7,1 \\
\hline 43. Comunicação prejudicada & 01 & 7,1 \\
\hline 44. Interação social prejudicada & 01 & 7,1 \\
\hline $\begin{array}{l}\text { 45. Nutrição alterada: ingestão maior que as } \\
\text { necessidades comorais }\end{array}$ & 01 & 7,1 \\
\hline 46. Diarréia & 01 & 7,1 \\
\hline 47. Incontinência intestinal & 01 & 7,1 \\
\hline 48. Padrão de sexualidade alterado & 01 & 7,1 \\
\hline 49. Perfusão tissular alterada & 01 & 7,1 \\
\hline 50. Débito cardíado diminuido & 01 & 7,1 \\
\hline
\end{tabular}


Conforme a Tabela 2, 16 diagnósticos de enfermagem obtiveram freqüências iguais ou superiores à 50\%, foram eles: Proteção alterada $(100 \%)$, Déficit de conhecimento (100\%), Alto risco para infecção $(85,7 \%)$, Integridade da pele prejudicada $(71,4 \%)$, Sensopercepção alterada $(71,4 \%)$, Distúrbio no padrão de sono $(71,4 \%)$, Dor $(71,4 \%)$, Alto risco para ferimentos $(64,3 \%)$, Excesso do volume de líquidos $(64,3 \%)$, Estratégias ineficazes de resolução individual $(57,1 \%)$, Nutrição alterada: ingestão menor que as necessidades corporais $(57,1 \%)$, Mucosa oral alterada $(50,0 \%)$, Ansiedade (50,0\%), Medo (50,0\%), Intolerância à atividade $(50,0 \%)$ e Déficit de lazer (50,0\%).

O diagnóstico Proteção Alterada é definido pela NANDA como "estado no qual o indivíduo tem diminuição da capacidade de defender-se de ameaças internas ou externas, tais como a doença ou injúria"14,15, sendo identificado em $100 \%$ da amostra. As alterações na imunidade, no processo de cicatrização tecidual e na coagulação foram características que fundamentaram esse diagnóstico. Na Tabela 3 estão representados os fatores causais ligados a este diagnóstico, e a freqüência de cada um deles na amostra estudada.

Tabela 3 - Freqüência de cada um dos fatores relacionados para o diagnóstico proteção alterada, nos clientes da amostra $(\mathrm{N}=14)$

\begin{tabular}{lcc}
\hline Fatores relacionados & $\mathbf{n}^{\mathbf{0}}$ & $\mathbf{\%}$ \\
\hline - Perfil sangüineo alterado & 14 & 100,0 \\
- Doença de base (doenças como câncer e & 14 & 100,0 \\
problemas imunológicos) & & \\
- Terapia medicamentosa & 12 & 85,7 \\
- Nutrição inadequada & 09 & 64,3 \\
- Extremo de idade & 03 & 21,4 \\
\hline
\end{tabular}

Tendo em vista as manifestações clínicas comuns em clientes portadores de doenças hematológicas (como cicatrização prejudicada, imunidade insuficiente, coagulação alterada) já era de se esperar que as causas apontadas na Tabela 3 (perfil sangüíneo alterado, doença de base, terapia medicamentosa, nutrição inadequada e extremo de idade) fossem as responsáveis por tais evidências clínicas. Os problemas advindos da doença hematológica podem significar dificuldades do organismo em se proteger de ameaças externas e internas.

Outro diagnóstico de enfermagem identificado em $100 \%$ da amostra foi Déficit de conhecimento, ligado ao processo de doença e/ou tratamento. Os fatores relacionados envolveram dificuldade de memorização, em decorrência das alterações de perfusão e dos componentes sangüíneos, bem como limitações cognitivas e falta de interesse. Vários estudos têm apontado para a grande incidência deste diagnóstico em diversas clientelas ${ }^{10,18}$, o que indica a necessidade do enfermeiro estar atento para o estabelecimento de estratégias que solucionem o problema.

É fundamental que o cliente compreenda o processo de cuidado, para que possa identificar sinais e sintomas da doença, maneiras de minimizar riscos, formas de prevenir complicações e medidas de promoção da saúde, especialmente em se tratando de clientes que apresentam doenças hematológicas.

Os diagnósticos classificados como potenciais, são aqueles que podem vir a ocorrer caso não sejam tomadas algumas medidas ${ }^{1}$. Na amostra estudada foram detectados dois diagnósticos nessa categoria: Alto risco para infecção, com uma freqüência de $85,7 \%$ e Alto risco para ferimentos, com frequiência de $64,3 \%$. Os fatores de risco identificados para os dois diagnósticos supracitados estão apresentados nas Tabelas 4 e 5, respectivamente.

Tabela 4 - Freqüência de cada um dos fatores de risco para o diagnóstico alto risco para infecção, nos clientes da amostra $(\mathrm{N}=12)$

\begin{tabular}{lcc}
\hline Fatores de riscos & $\mathbf{n}^{\mathbf{0}}$ & $\mathbf{\%}$ \\
\hline - Defesa secundária inadequada & 12 & 100,0 \\
(alteraçóes na crase sangǘnea) & 11 & 91,7 \\
- Doença crónica & 10 & 83,3 \\
- Procedimentos invasivos & 10 & 83,3 \\
- Uso de agentes medicamentosos & 09 & 75,0 \\
- Defesa primária inade quada (te cidos & & \\
traumatizados) & 06 & 50,0 \\
- Hospitalizaçăo prolongada
\end{tabular}

Estes resultados estão em consonância com a literatura ${ }^{20}$, sendo de certa forma esperados, pois relacionam-se às alterações advindas da doença hematológica (imunossupressão, leucopenia, anemia), bem como às consequiências de uma doença que exige tempo de internação prolongado, utilização de procedimentos invasivos e tratamentos com agentes medicamentosos (antibióticos e quimioterápicos). Cabe ressaltar o fator de risco "defesa primária inadequada", presente em $75 \%$ da amostra, pois havendo injúria e lesão de continuidade da pele, associado às alterações hematológicas, aumentam os riscos para as infecções.

Tabela 5 - Frequiência de cada um dos fatores de risco para o diagnóstico Alto risco para ferimentos, nos clientes da amostra $(\mathrm{N}=09)$

\begin{tabular}{lcc}
\hline Fatores de risco & $\mathbf{n}^{\mathbf{0}}$ & $\mathbf{\%}$ \\
\hline - Perfil sangüineo anormal & 09 & 100,0 \\
- Agentes farmacêuticos & 07 & 77,8 \\
- Doenca crônica & 07 & 77,8 \\
- Disfunção sensorial & 05 & 55,5 \\
- Alterações na mobilidade & 05 & 55,5 \\
- Lesões de pele & 04 & 44,4 \\
- Idade avançada & 03 & 33,3 \\
- Transporte em cadeiras e com aparelhos & 03 & 33,3 \\
- Má nutrição & 02 & 22,2 \\
- Uso de produtos químicos no domicilio & 02 & 22,2 \\
- Presença de convulsões & 01 & 11,1 \\
\hline
\end{tabular}


Na Tabela 5 podem-se identificar os fatores de risco da amostra estudada, relativos ao diagnóstico Alto risco para ferimentos. Os fatores "perfil sangüíneo anormal" e "uso de agentes farmacêuticos" estiveram presentes na maioria dos clientes com o diagnóstico em questão, corroborando com dados da literatura relativa à clientela. A hipóxia, a leucocitose e a leucopenia, são distúrbios representativos de anormalidades hematológicas (evidenciados em patologias como as Anemias, Leucemias, Linfomas e Mielomas) que conhecidamente (decorrentes das alterações no perfil sangüíneo) modificam os processos de hemostasia e oxigenação tecidual, bem como, os sistemas de defesa, contribuindo assim, para o alto risco de ferimentos a que esta clientela está sujeita.

Ainda, com relação ao diagnóstico Alto risco para ferimentos, vários fatores encontrados na clientela estudada, estão associados à própria doença de base. As alterações na percepção sensorial (Disfunção sensorial), presentes em 55,5\% dos clientes da amostra - conforme Tabela 5, identificadas como dificuldades visuais e táteis, podem ser responsáveis por uma série de traumatismos e ferimentos no cliente com doença hematológica, assim como Alterações na mobilidade (presentes em 55,5\% dos clientes da amostra) e Transporte em cadeiras e com aparelhos (presentes em 33,3\% dos clientes da amostra), dentre outros.

ULRICH et al. ${ }^{20}$ apontam o diagnóstico Alto risco para ferimentos como sendo de alta probabilidade de ocorrência em clientes com doenças hematológicas.

$\mathrm{Na}$ amostra estudada, 10 clientes $(71,4 \%)$ apresentaram sinais de destruição das camadas da pele e solução de continuidade, caracterizando o diagnóstico Integridade da pele prejudicada. Dentre os fatores causais, foram identificados: déficit imunológico (em $70 \%$ dos clientes com esse diagnóstico), alteração no estado metabólico (infecções e tumor, em 60\%), alteração na mobilidade física (50\%), fatores mecânicos (uso de adesivos, traumas por punções venosas, proeminências ósseas sem proteção e injúria pelo tumor, em 50\%), sensibilidade e circulação alteradas ( $40 \%$ em cada uma), mudanças no estado nutricional $(30 \%)$ e substância química (infiltração de drogas quimioterápicas, em 10\%).

Sabe-se que, em decorrência de fatores internos ligados à própria doença (como alterações nos processos de cicatrização e reprodução celular, defesa, circulação e oxigenação dos tecidos), os clientes com alterações hematológicas têm alta probabilidade de apresentar o diagnóstico Integridade da pele prejudicada. Além disso, fatores externos (como a contínua necessidade de realização de procedimentos invasivos e o uso de medicações quimioterápicas), podem estar presentes. $\mathrm{Na}$ amostra estudada foram detectados fatores causais, externos e internos, para o referido diagnóstico, que em associação contribuíram para sua evidência.
O diagnóstico Senso-percepção alterada esteve presente em $71,4 \%$ da amostra. É definido como "estado no qual o indivíduo experimenta uma mudança na quantidade ou no tipo de estímulos percebidos, acompanhada por uma resposta diminuída, exagerada, distorcida ou enfraquecida a tais estímulos" ${ }^{\text {"14,15. }}$.

Nos 10 clientes com este diagnóstico, estiveram associados déficit nos sentidos da visão $(80,0 \%)$, audição $(40,0 \%)$, tato $(40,0 \%)$, paladar $(10,0 \%)$ e da percepção dos movimentos $(10,0 \%)$.

Os fatores causais do diagnóstico Sensopercepção alterada, presentes na amostra, relacionaramse, em grande parte, aos distúrbios de sangramento a nível central, como ocorreu em dois clientes com anemia aplásica, e aos danos de tecidos tumorais à inervação periférica. Outros aspectos relevantes, responsáveis pelos sinais e sintomas do diagnóstico Alteração na percepção sensorial, não estavam relacionados diretamente à doença de base em alguns clientes, podendo estar, em parte, ligados às modificações decorrentes do processo de maturescência.

Foram explicitadas, pelos clientes do presente estudo, dificuldades para adormecer e sono interrompido, fundamentando o diagnóstico Distúrbio no padrão do

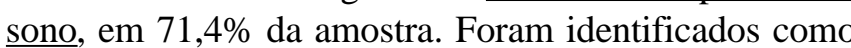
fatores relacionados as alterações sensoriais internas (estresse psicológico, dores, febre noturna e inatividade), em $100 \%$ dos clientes que apresentaram este diagnóstico.

As alterações externas (como barulho do ambiente, luminosidade e interrupção do sono para a verificação dos sinais vitais), foram detectadas em 30,0\% dos clientes que apresentaram o diagnóstico em questão, associadas às alterações internas já descritas.

O cliente com doença hematológica está vulnerável a desordens no padrão de sono devido a razões ligadas ao aumento da taxa metabólica, alterações na rotina e nos padrões usuais de dormir ou deitar, ansiedade, depressão, estresse, inatividade, e outras.

Alguns destes fatores relacionados descritos para o diagnóstico Distúrbio no padrão de sono, estiveram presentes no diagnóstico Déficit de lazer, e ambos estão, de certa forma, muito relacionados. A inatividade durante o período diurno, por falta de opções ou interesse no lazer, causará dificuldades para o repouso noturno adequado.

$\mathrm{Na}$ amostra estudada, $50,0 \%$ dos sujeitos experimentaram uma diminuição no interesse ou no engajamento em atividades recreativas, formulando-se assim o diagnóstico Déficit de lazer. Dentre as causas identificadas podemos citar as relativas ao tédio por internações prolongadas (ocorrido em $85,7 \%$ dos clientes que apresentaram o diagnóstico Déficit de lazer), dificuldade de engajamento em atividades devido limitações físicas (em 71,4\% destes clientes) e estado psicológico de depressão (em 28,6\% dos clientes). 
Outro diagnóstico de enfermagem que apresentou alta frequiência na amostra estudada, foi o diagnóstico de Dor $(71,4 \%)$, tendo como características definidoras a comunicação verbal da dor e a presença de comportamentos expressivos de desconforto (mudanças de posição, inquietação e fácies de dor).

Os fatores causais identificados na amostra, para o diagnóstico de Dor, foram: injúria dos tecidos por agentes biológicos (infecções em 30,0\% dos clientes que apresentaram este diagnóstico) e mecânicos (hematoma, tumor ou edema, totalizando 70,0\% destes clientes). Os agentes químicos também tiveram, em associação aos mecânicos, freqüência detectada em $10,0 \%$ destes clientes.

Como pode se observar na Tabela 2, o diagnóstico de enfermagem Excesso do volume de líquidos ocorreu em $64,3 \%$ dos clientes da amostra, estando caracterizado pela presença de edema. As causas estavam relacionadas ao decréscimo de proteínas plasmáticas (em 55,5\% dos clientes com o referido diagnóstico), obstrução linfática devido à compressão tumoral (em 22,2\%), aumento da permeabilidade capilar por trauma cirúrgico (em 11,1\%) e nefrite por irradiação (em 11,1\%).

A alta freqüência do diagnóstico Excesso do volume de líquidos identificada neste estudo está de acordo com a literatura geral, uma vez que, indivíduos com doenças hematológicas estão suscetíveis a alterações no equilíbrio hídrico.

No que se refere ao diagnóstico de enfermagem Estratégias ineficazes de resolução individual, a frequiência foi de $57,1 \%$, na amostra estudada.

Este diagnóstico é definido como "estado no qual o indivíduo demonstra dificuldade para apresentar comportamentos adaptativos e habilidade na resolução de problemas para atender as demandas e os papéis da vida" ${ }^{14,15}$.

A doença neoplásica e seu tratamento trazem problemas psicológicos diversos ao cliente, a gravidade e a duração destes problemas estão fortemente influenciados pelos mecanismos de enfrentamento (estratégias) por ele utilizados. Tais estratégias poderão ser efetivas ou ineficazes, conscientes ou inconscientes ${ }^{13}$.

As estratégias ineficazes são mal sucedidas e o cliente pode apresentar isolamento, projeção (culpar alguém ou alguma coisa), depressão, supressão (tentar retirar o problema da mente), somatização, e outros ${ }^{6}$.

Nos clientes deste estudo com o diagnóstico em questão, foram identificados sintomatologias variáveis que envolveram a demonstração da incapacidade para resolução dos problemas pessoais, incapacidade de alcançar e desempenhar papéis desejáveis, mudanças na participação social e uso inadequado dos mecanismos de defesa, todos expressos sob a forma de raiva e hostilidade, isolamento, depressão, negação e supressão.
Cabe ressaltar que dos 08 clientes da amostra que apresentaram o diagnóstico de enfermagem Estratégias ineficazes de resolução individual, 03 (37,5\%) desenvolveram o referido diagnóstico com o progredir do período de internação, quando as estratégias utilizadas passaram a ser ineficazes e o indivíduo passou a vivenciar a crise (entendida aqui como um período de desequilíbrio psicológico, onde o indivíduo, frente a um problema importante ou obstáculo, é incapaz de utilizar eficazmente as estratégias de enfrentamento ${ }^{3}$ ). Sinais e sintomas como dificuldades para dormir, aumento da fadiga, irritabilidade, dificuldades de concentração, decréscimo da tolerância à dor e dificuldades para resolução de problemas, estiveram presentes nestes clientes e estes dados vão ao encontro dos relatos de ULRICH et al. ${ }^{20}$ sobre os sinais e sintomas que indicam coping ineficaz.

Quanto os fatores causais envolvidos, como pode ser visto na Tabela 6 , todos os clientes com o diagnóstico em questão apresentaram crise situacional.

Tabela 6 - Freqüiência de cada um dos fatores relacionados para o diagnóstico Estratégias ineficazes de resolução individual, nos clientes da amostra $(\mathrm{N}=08)$

\begin{tabular}{lcc}
\hline Fatores relacionados & $\mathbf{n}^{\mathbf{0}}$ & $\mathbf{0}$ \\
\hline - Crise situacional & 08 & 100,0 \\
- Conflitos por mudanças no estilo de vida & 07 & 87,5 \\
- Vulnerabilidade pessoal & 06 & 75,0 \\
- Expectativas não atingidas & 04 & 50,0 \\
- Relaxamento inadequado & 03 & 37,5 \\
- Crise existencial (adolescência) & 01 & 12,5 \\
- Perspectivas não realistas & 01 & 12,5 \\
\hline
\end{tabular}

A doença hematológica provoca desequilíbrios no cliente de toda ordem. As hospitalizações constantes e prolongadas, as mudanças decorrentes do agravamento no quadro, a freqüente ameaça de morte ou sofrimento, as alterações na imagem corporal, a separação e o afastamento do convívio com familiares, as modificações na habilidade de desempenhar papéis, os possíveis gastos adicionais com o tratamento e as ameaças de perda de emprego, são todos fatores desencadeantes para este desequilíbrio.

Dois diagnósticos de enfermagem, também detectados na amostra estudada, que estão em íntima relação com a discussão apresentada, são os de Ansiedade e_Medo, ambos presentes em 50,0\% dos clientes do estudo.

Para o diagnóstico Nutrição alterada: ingestão menor que as necessidades corporais, presente em 57,1\% da amostra, foram identificados variados sinais e sintomas como: emagrecimento (peso corporal abaixo de 20\%), diminuição da massa muscular, relato de ingestão 
inadequada de alimentos por dificuldades na aceitação da dieta e inabilidade para a deglutição. Dentre os fatores causais para este diagnóstico, houve predominância dos biológicos, que, presentes em associação, destacaramse: a inapetência e o hipermetabolismo (presentes, cada um, em $62,5 \%$ dos clientes com o diagnóstico em questão), as náuseas e os vômitos (presentes em 12,5\% dos clientes) e a dificuldade de deglutição e mastigação (presentes em 25,0\% dos clientes). Os fatores psicológicos identificados, associados aos biológicos, estiveram ligados à depressão e ao desânimo (em 50,0\% dos clientes), bem como à associação negativa dos alimentos à quimioterapia (em $12,5 \%$ dos clientes).

McGUIGAN \& WOLFE ${ }^{12}$ relatam ser freqüente a perda de peso, decorrente das associações como anorexia, disfagia, náuseas e vômitos, em clientes hospitalizados ou com patologias crônicas consumptivas. Esta afirmação concorda com os achados do presente estudo.

Cabe ressaltar a importância das doenças hematológicas neoplásicas na contribuição da perda de massa corporal, em função das alterações no metabolismo de proteínas, gorduras e carboidratos, levando à caquexia característica destes clientes.

Não menos importantes, e também relacionadas às alterações na ingesta alimentar e perda de peso, estão as lesões de orofaringe, comuns aos clientes imunodeprimidos, como nos indivíduos com alterações hematológicas. No presente estudo, as lesões de orofaringe estiveram presentes em 71,4\% dos clientes, confirmando o diagnóstico de enfermagem Mucosa oral alterada.

Além das lesões de orofaringe, o diagnóstico Mucosa oral alterada, contou com outros sinais como a presença de cáries e placas bacterianas em 100,0\% dos clientes com o referido diagnóstico, refletindo a necessidade do enfermeiro estar atento para o problema. Assim, é importante que sejam estabelecidas estratégias, junto à essa clientela, que permitam a adequada orientação quanto a realização da higiene oral e a supervisão constante das condições de higiene e integridade da boca.

Ainda, como pode ser observado na Tabela 2, o diagnóstico de enfermagem Intolerância à atividade esteve presente em 50,0\% da amostra estudada, tendo como sintomatologia, em 100,0\% dos clientes com este diagnóstico, o relato de "fraqueza generalizada", fadiga ou desânimo. Dentre os fatores etiológicos identificados, conforme apresentado na Tabela 7, os mais expressivos estiveram relacionados à doença crônica ou neoplásica (em 100,0\% da amostra com o diagnóstico em questão), o repouso prolongado ou a imobilidade e as desordens nutricionais (em 42,8\%, cada um).
Tabela 7 - Frequiência de cada um dos fatores relacionados para o diagnóstico Intolerância à atividade, nos clientes da amostra $(\mathrm{N}=07)$

\begin{tabular}{lcc}
\hline Fatores relacionados & $\mathbf{n}^{\mathbf{0}}$ & $\mathbf{\%}$ \\
\hline - Doença crônica ou neoplásica & 07 & 100,0 \\
- Repouso prolongado no leito ou mobilidade & 03 & 42,8 \\
- Desordens nutricionais & 03 & 42,8 \\
- Desequilibrio entre suprimento e demanda & 01 & 14,3 \\
de oxigênio & & \\
- Depressão & 01 & 14,3 \\
- Estágio desenvolvimental & 01 & 14,3 \\
\hline
\end{tabular}

Ressalta-se a íntima associação dos aspectos ligados a mobilidade física com o diagnóstico Intolerância à atividade.

Os clientes com distúrbios na crase sangüínea, por apresentarem uma doença consumptiva, estão propensos a sofrer uma diminuição de energia, em virtude, basicamente, de fatores relacionados às alterações da constituição sangüínea e das atividades metabólicas. A anemia (seja devido aos desencadeamentos da própria doença, seja pelo uso de quimioterápicos), o estado nutricional deficitário, o estado emocional alterado (como a depressão), as restrições impostas pela terapia, bem como as alterações sensoriais e motoras, são responsáveis por gerar fraqueza, dispnéia, desequilíbrios no suprimento de oxigênio e alterações na atividade e mobilidade física. Sendo assim, os achados do presente estudo estão de acordo com os dados da literatura relativos à clientela em foco.

\section{CONSIDERAÇÕES FINAIS}

A etapa do diagnóstico de enfermagem tem sido objeto de investigação e vem representando um dos principais passos da sistematização da assistência de enfermagem, por contribuir para a identificação dos problemas do cliente e posterior formulação das intervenções de enfermagem ${ }^{7}$.

Para a clientela estudada (14 sujeitos) foram arrolados 240 diagnósticos de enfermagem, sendo a frequiência média de 16,9 diagnósticos por cliente. Estes diagnósticos caracterizaram-se em 50 diferentes tipos, dentre os padronizados pela NANDA na Taxonomia $I^{15}$.

Destes, 16 diagnósticos apresentaram alta frequiência na amostra estudada (frequiência acima ou igual a 50\%), sendo eles: Proteção alterada (100\%), Déficit de conhecimento (100\%), Alto risco para infecção $(85,7 \%)$, Integridade da pele prejudicada $(71,4 \%)$, Sensopercepção alterada $(71,4 \%)$, Distúrbio no padrão de sono $(71,4 \%)$, Dor $(71,4 \%)$, Alto risco para ferimentos 
$(64,3 \%)$, Excesso do volume de líquidos $(64,3 \%)$, Estratégias ineficazes de resolução individual $(57,1 \%)$, Nutrição alterada: ingestão menor que as necessidades corporais $(57,1 \%)$, Mucosa oral alterada $(50,0 \%)$, Ansiedade (50,0\%), Medo (50,0\%), Intolerância à atividade $(50,0 \%)$ e Déficit de lazer $(50,0 \%)$.

Estes, bem como os demais diagnósticos identificados, apresentados na Tabela 2, apresentam relação com as patologias hematológicas observadas na clientela, sobretudo Mielomas, Linfomas, Anemias e Leucemias.

Quanto às variáveis sexo e idade, na amostra estudada, houve predominância de indivíduos adultos, com distribuição ligeiramente maior para o sexo masculino, refletindo os aspectos epidemiológicos habituais das doenças hematológicas apresentadas pelos clientes estudados.

Cabe ressaltar a necessidade de outros estudos nesta área, junto a clientes com alterações hematológicas, para a definição de perfil diagnóstico, a fim de subsidiar a assistência de enfermagem sistematizada e fundamentada cientificamente.

Contudo, para que o enfermeiro possa identificar perfis diagnósticos de diferentes clientelas, é fundamental que domine o processo de julgamento clínico. Nesse processo, o enfermeiro avalia o significado das informações sobre o cliente estabelece relações entre os dados, nomeia o fenômeno e chega ao diagnóstico a partir da inferência e de estratégias de pensamento, com base em suas experiências, conhecimentos e valores. Requer habilidades de manipulação dos dados, domínio de conhecimento, aptidão intelectual, objetividade, pensamento crítico, experiência, intuição e tomada de decisão.

Assim sendo, destaca-se a complexidade e extensão dos conhecimentos necessários para que o enfermeiro realize o julgamento clínico e formule diagnósticos de enfermagem.

\section{NURSING DIAGNOSES IN CLIENTS WITH HEMATOLOGIC DISEASES: USE OF NANDA'S TAXONOMY I}

The present paper aims to describe the most frequent nursing diagnoses found in a group of clients with hematologic diseases. Authors used the Taxonomy I proposed by the North American Nursing Diagnoses Association (NANDA) to guide this study. Data were collected from 14 clients. Authors identified 16 nursing diagnoses whose frequency were equal or higher to fifty percent. Other studies are needed to find a better definition of the diagnostic profile of these clients.

\section{DIAGNÓSTICOS DE ENFERMERÍA EN CLIENTES CON ALTERACIONES HEMATOLÓGICAS: USO DE LA TAXONOMÍA I DE LA NANDA}

El presente estudio visa describir los más frecuentes diagnósticos de enfermería en un grupo de clientes portadores de enfermedades henatológicas. Fue utilizada la Taxonomia I, propuesta por la North American Nursing Diagnosis (NANDA), para el direccionamiento de la investigatión. Fueron analizados los datos colectados de 14 clientes, siendo identificados 16 diagnósticos de enfermería con frecuencia igual o superior a 50,0\%. Se recomienda la realización de otros estudios para una mejor definición del perfil diagnosticado en esta clientela.

TÉRMINOS CLAVES: diagnóstico de enfermería, proceso de enfermería, cliente hemotológico

\section{REFERÊNCIAS BIBLIOGRÁFICAS}

01. CARPENITO, L. J. Nursing diagnosis: application to clinical practice. Philadelphia: J. B. Lippincott, 1987. p. 2-86.

02. CARROLL-JOHNSON, R. M.; PAQUETTE, M. Classification of nursing diagnosis: proceedings of the tenth conference. Philadelphia: J. B. Lippincott, 1994. p.421-22.

03. CRAIG, H. M.; EDWARDS, J. E. Adaptation in chronic illness: an eclect model for nurses. J. Adv. Nurs., v. 8, p. 397-404, 1983.
04. CRUZ, D. de A. L. M. da Os diagnósticos de enfermagem no ensino e na pesquisa. Rev. Esc. Enfermagem USP, v. 26, n. 3, p. 427-434, dez. 1992.

05. CRUZ, I. C. F. da et al. Estudo descritivo sobre as características definidoras do diagnóstico de enfermagem: disfunção sexual. Rev. Esc. Enfermagem USP, v.26, n.1, p. 43-64, mar. 1992.

06. DAVIS, D. S. Common clinical problems: coping. In: JOHNSON, B. L. ; GROSS, J. Handbook of oncology nursing. New York: John Wiley, 1985. p. $129-144$. 
07. EDEL, M. The nature of nursing diagnosis. In: CARLSON, J. et al. Nursing diagnosis. Philadelphia: W. B. Saunders, 1982. p.1-17.

08. GORDON, M. Nursing diagnosis: process and application. 3.ed. St Louis: Mosby, 1994. cap. 1, p. 1-16.

09. JESUS, C. A. C. de Assistência de enfermagem a clientes hematológicos: uma visão sistêmica. Ribeirão Preto, 1992. 279p. Dissertação (Mestrado) - Escola de Enfermagem de Ribeirão Preto, Universidade de São Paulo.

10. KAMIYAMA, Y. O doente hospitalizado e sua percepção quanto à prioridade de seus problemas. São Paulo, 1972. 111p. Tese (Doutorado) - Escola de Enfermagem, Universidade de São Paulo.

11. MANZOLLI, M. C. Relacionamento em enfermagem: aspectos psicológicos. São Paulo: Sarvier, 1987. p. 49-58.

12. McGUIGAN, J. E.; WOLFE, M. M. Anorexia, náuseas e vômitos. In: BLACKLOW, R. S. MacBryde sinais e sintomas: fisiopatologia aplicada e interpretação clínica. Rio de Janeiro: Guanabara Koogan, 1986. p. 342-353.

13. MEYEROWITZ, B. E. et al. A competency-based approach to coping with cancer. In: BURISH, T. G. et. al. Coping with chronic disease: research and applications. New York: Academic Press, 1983. p. 137-189.
14. NÓBREGA, M. M. L. da; GARCIA, T. R. (org.) et al. Uniformização da linguagem dos diagnósticos de enfermagem da NANDA: sistematização das propostas do II SNDE. João Pessoa: A União, CNRDE/GIDE-PB, 1994. $138 \mathrm{p}$.

15. NORTH AMERICAN NURSING DIAGNOSIS ASSOCIATION. Taxonomia I Revised with official nursing diagnosis. St. Louis, 1990. $127 \mathrm{p}$.

16. PIMENTA, C. A. M. et al. O ensino da avaliação do paciente: delineamento do conteúdo pelo diagnóstico de enfermagem. Rev. Latino-Am. Enfermagem, v. 2, n.1, p. 69-76, Julho 1993.

17. POTTER, P. A.; PERRY, A. G. Fundamentals of nursing. Saint Louis: C. V. Mosby, 1989. cap. 6, p. 102-121.

18. RICCIO, G. M. G. et al. Diagnóstico de enfermagem dos pacientes submetidos a terapêutica trombolítica. Rev. Soc. Cardiol. Estado de São Paulo, v. 4, n. 3, p. 6-16, Mai/ Jun. 1994.

19. THOMAS, N. M.; NEWSOME, G. G. Factors affeting the use of nursing diagnosis. Nurs. Outlook, v. 40, n. 4, p. 182-186, July/August. 1992.

20. ULRICH, S. P. et al. Nursing care planning guides: a nursing diagnosis approach. Philadelphia: W. B. Saunders, 1986. p. 441-467. 